\title{
Simulation of seismic response in an idealized city
}

\author{
Chrysoula Tsogka, Armand Wirgin \\ Laboratoire de Mécanique et d'Acoustique, UPR 7051 du CNRS, 31 chemin Joseph Aiguier, 13402 \\ Marseille cedex 20, France. \\ We study the seismic response of an idealized 2D 'city', constituted by ten non equally- \\ spaced, non equally-sized, homogenized blocks (i.e., buildings or groups of buildings) \\ anchored in a soft soil layer overlying a hard halfspace. Our results display strong \\ response inside the blocks and on the ground which qualitatively match the responses \\ observed in some earthquake-prone cities.
}

Keywords: earthquakes, cities, beating, amplification and long coda of vibrations

A noticeable feature of many earthquake-prone cities such as Kobe ${ }^{1}$ (Japan), Izmit ${ }^{2}$ (Turkey), Nice ${ }^{3}$ (France), Mexico City ${ }^{4-6}$, Los Angeles ${ }^{7-10}$ etc., is that they are partially or wholly built on soft soil. A straightforward 1D analysis ${ }^{11}$ (which takes no account of the presence of the buildings) shows that the soft layer increases the seismic vulnerability of the city in that it is responsible for amplification of ground motion during an earthquake. However, the 1D model does not account either for the beating phenomena and very long codas in the building vibrations, nor for the large spatial variability of response, repeatedly observed in sites such as Mexico City ${ }^{5,6}$.

To analyze the possible causes of these puzzling effects, we study the action of a seismic wave on a relatively-simple structural model with both geological and man-made features. Our 2D model has three components (from bottom to top in Fig.1): a hard half space (HHS), overlaid by a soft soil layer (SL), in which are partially imbedded a set of even softer blocks (SB). HHS and SL are geological features, the set of SB, which are homogenized for the purpose of the analysis (see fig.2), is man-made and constitutes the 'visible' component of an idealized city.

First consider the case in which the blocks are absent. Then the stress-free surface (upper boundary of the soft layer constituting the ground) is a straight horizontal line (in Fig.

\footnotetext{
${ }^{1}$ E-mail : wirgin@1ma.cnrs-mrs.fr, tel. : 334911640 50, fax 33491220875.
} 
3), so that the configuration is the one to which the $1 \mathrm{D}$ analysis of the problem applies. In the SH polarization case, the modes of this $1 \mathrm{D}$ configuration are Love waves ${ }^{12-14}$ which are only weakly excited by a teleseismic source and not at all by an incident plane seismic wave ${ }^{12,13}$. If, however, the HHS/SL interface is irregular (curved, rough) or the lateral extent of the SL is finite, the boundary irregularities or edges of the SL become diffractors, enabling substantial exitation of so-called quasi-Love modes (this term is employed when the HHS/SL interface is no longer a horizontal straight line). The result of this is that the field in the SL and on the ground becomes amplified (often more so than in the 1D model) at certain resonance frequencies (which are different from those of the Love modes) ${ }^{13-18}$.

If the city is viewed as a non-interacting building/substratum system, the building motion is considered separately from, and to not influence, the motion of the substratum. The motion of the substratum (the latter can even be considered to be a quite complex structure e $^{3,7-}$ ${ }^{11}$ ) is, in fact, viewed as being transmitted as such to the buildings by a forced vibration mechanism. The building motion is large when the (low) resonance frequencies of the building coincide with the (low) resonance frequencies of the substratum ${ }^{19}$. When there are several buildings in the city, they are usually thought to act independently of one another, and the ones that exhibit the largest motion are those whose (low) resonance frequencies coincide with the (low) resonant frequency of the substratum. The translation of this in the time domain is that some buildings will have motion: which is more ample than that of the ground (in the absence of the buildings), which varies from building to building (if these are different from one another), and which may last longer than that of the ground (in the absence of the buildings). Grossly speaking, this is what has been observed in the above-mentioned cities, so that the paradigm we have just described is the one that is usually implicit in attempts $3,5-10,19$ to describe and understand what is likely to happen when a seismic wave hits a city built on soft soil.

Now suppose that the substratum (especially its irregularities) of the city is not known other than by the fact that it is thought to consist of a soft component of approximate (or average) thickness $\tau$, wherein the density and shear wavespeed are approximately (or on the average) $\rho$ and $c$ respectively, overlying hard rock. To analyze the seismic response of this configuration, it would probably be most reasonable to model the latter as a horizontal constant-thickness layer with characteristic parameters $\tau, \rho$ and $c$ overlying hard rock, but unfortunately, this gives rise (as mentioned in our introductory phrases) to a response that may be quite different from the observed response in urban sites such as Mexico City. 
However, there is an aspect of the city that has not yet been taken into account and which is quite 'visible': the set of buildings (with foundations) of which it is composed. It is not unreasonable to expect these buildings, together with their foundations, to act in a manner similar to that of the previously-mentioned diffractors, thereby exciting quasi-Love waves (for SH polarization) which, assuming the substratum to still be of the 1D type, may lead, in the way described above, to shaking that is stronger, and of longer duration, in the buildings than on the gound (in the absence of the buildings) ${ }^{20-28}$, and vary from building to building if the latter differ from each other and have non-constant spacing. The purpose of this work is to demonstrate this fact. To do so, we solve the governing equations of linear elastodynamics relative to the complete configuration, i.e., the homogenized blocks together with the substratum. Note that this approach is different from the one used in publications such as Refs. 19,20,24,28, wherein the buildings+foundations are considered either to be shear walls with rigid or flexible bases, or inverted pendulums attached to mass/spring/dashpot systems.

\section{Basic ingredients of our approach}

Referring once again to Fig.1, the blocks of the city are connected to the substratum by an interface on which we assume the continuity of displacement and normal stress. These conditions are implicit when the heterogeneous medium, constituted by the half-space underneath the free surface, is considered to be a continuum. The governing equations, with or without the blocks, are those of linear elastodynamics for a heterogeneous, isotropic medium with upper stress-free boundary. When the blocks are present, the formerly-flat ground becomes the portions of the ground in between the blocks plus the boundaries of the blocks in contact with the air. In principle, it is not more difficult to solve numerically these equations for the configuration with blocks than the configuration without blocks. Of course, the computational effort is increased due to the additional degrees of freedom needed to model the motion of the blocks. The increase in computational effort is similar to the one that arises when the ground is flat and the substratum is considered to have some small-scale structure, such as in the most recent studies ${ }^{7-10}$.

Our 'city' is invariant in the $x_{3}$-direction with $x_{1}, x_{2}, x_{3}$ being the cartesian coordinates, and $x_{2}$ increasing with depth (see Fig. 3 wherein the $x_{1}-x_{2}$ sagittal plane is displayed). The seismic source is a line in the $x_{3}$-direction, and located deep in the HHS, radiating a Ricker pulse cylindrical shear-horizontal $(\mathrm{SH})$ displacement field. Only the $x_{3}$ - 
component of this field is non-vanishing and invariant with respect to $x_{3}$, so that the total field underneath and on the free surface is also $S H$-polarized and invariant with respect to $x_{3}$. The resulting problem is now $2 \mathrm{D}$ with the displacement field depending only on $x_{1}, x_{2}$ and on time $t$. In Fig. 3 we denote by $h, w$ and $d$, the height, width and space interval between blocks. Contrary to what is assumed in the computations of Refs. 21,22,25, we consider $h, w$ and $d$ to vary from one block to another, as is often the case in real-life cities. The half-space underneath the irregular stress-free surface is occupied by a linear, isotropic, heterogeneous medium, characterized by mass density $\rho(\mathbf{x})$ and shear modulus $\mu(\mathbf{x})$, with $\mathbf{x}=\left(x_{1}, x_{2}\right)$. Both $\rho(\mathbf{x})$ and $\mu(\mathbf{x})$ are considered to be positive real, piecewise constant, time-invariant functions.

In addition to the configuration being 2D, no intrinsic medium losses are taken into account. Furthermore, the blocks are considered to be homogeneous, this meaning that their space-independent elastic parameters are chosen so that the average response in a given homogeneous block is approximately equal to that of the corresponding heterogeneous block (see fig. 2). This assumption was made in most previous studies of the seismic response of city-like configurations ${ }^{20-22,25-28}$. However, this homogenization procedure is not essential to our method of computation and will be modified in subsequent studies to take more precisely into account the structural detail of the blocks (and of the individual buildings of which they are composed). The impact of the no-loss assumption on the overall response is wellknown ${ }^{27}$ : both the intensity and duration of shaking in the buildings and on the ground is reduced in proportion to $Q^{-1}$, with $Q$ the quality factor which is infinite when the media are lossless. In Ref. 24, 2D models are considered to overestimate radiation damping and thus underestimate the intensity of ground motion, but there is no unanimous opinion about this issue. The only way to find out if this is true in the present case is to carry out the timedomain computations for a 3D city (with buildings included explicitly). This is presently beyond the state-of-art (at least with the computers at our disposal), although it has been done to some extent, with simplified models of building motion ${ }^{26,28}$. It is encouraging to note that the investigation in Ref. 28 leads to results (notably concerning the most important features of response) that are similar to those presented herein. However, the results of Ref. 26 are quite different from ours, perhaps due to the fact that the density and aspect ratios of buildings are unrealistically high and building/soil/building interactions are treated in approximate manner in this work. It is clear that, in order to establish the applicability of our results to real cities, it 
will be necessary to carry out a statistical analysis, which will evaluate the influence on the response of various distributions of block sizes and compositions, aspect ratios and separations, as well as of source types and locations and substratum geometries and compositions. Furthermore, 3D geometries, blocks decomposed into series of buildings, and realistic attenuations in the blocks and substratum will have to be taken into account in future studies.

\section{Methods}

The wave equation in linear, isotropic, heterogeneous solids is:

$$
(\lambda+\mu) u_{k, k l}+\mu u_{l, k k}+\lambda_{, l} u_{k, k}+\mu_{, k}\left(u_{k, l}+u_{l, k}\right)-\rho \partial_{t}^{2} u_{l}=-\rho f_{l} ; \quad l, k=1,2,3 \quad,
$$

wherein the Einstein summation convention is implicit and all symbols (i.e., $\lambda, \mu, \rho, u_{l}, f_{l}$ ) are functions of position, but only $u_{l}, f_{l}$ are functions of time. Note that $\lambda$ is the bulk modulus, $u_{l}$ the $l$-th cartesian component of particle displacement and $f_{l}$ the $l$-th cartesian component of applied force.

In the geometrical situation depicted in fig. 2, $S H$ waves correspond to $\phi_{, 3}=0$ (wherein $\phi=\lambda, \mu, \rho, u_{l}, f_{l}$ ) and

$$
u_{1}=u_{2}=f_{1}=f_{2}=0
$$

Consequently, (1) reduces to the equation

$$
\mu\left(u_{3,11}+u_{3,22}\right)+\mu_{, 1} u_{3,1}+\mu_{, 2} u_{3,2}-\rho \partial_{t}^{2} u_{3}=-\rho f_{3}
$$

whose vectorial expression is:

$$
\mu \nabla \cdot \nabla u_{3}+\nabla \mu \cdot \nabla u_{3}-\rho \partial_{t}^{2} u_{3}=-\rho f_{3}
$$

in which $\nabla \phi=\left(\phi_{, 1}, \phi_{, 2}\right)$.

This equation is second-order in terms of $t$ and an outcome of two equations, one of which expresses conservation of momentum, and the other is the constitutive relation of an isotropic, elastic medium. A rearrangement of these equations enables them to be cast into the form of the following two first-order-in-time equations: 


$$
\begin{gathered}
\partial_{t} \mathbf{v}+\mu \nabla u_{3}=0 \\
\rho \partial_{t} u_{3}+\nabla \cdot \mathbf{v}=\rho g .
\end{gathered}
$$

wherein $\partial_{t} \mathbf{v}$ is related to the non-zero components of the stress tensor. It is easy to verify, that taking the divergence of (5), the time derivative of (6), and subtracting the two equations so obtained, leads to (3) provided that

$$
f_{3}=\partial_{t} g
$$

The problem is to solve (3) for $\mathbf{x} \in \mathbf{R}^{2}$ and $t \in \mathbf{R}^{+}$subject to an initial condition, a radiation condition (the waves must be outgoing from the source and free surface boundary) and the free surface boundary condition

$$
\mu \mathbf{n} \cdot \nabla u_{3}=0 \text { on } \Gamma
$$

which expresses the fact that the normal component of stress vanishes on the interface $\Gamma$ between the city (portions of the boundaries of the blocks in contact with air, plus the ground in between blocks) and the ambient air (assumed to be a vacumn, with $\mathbf{n}$ the unit vector normal to $\Gamma$ ). Rather than deal with the single second-order-in-time scalar partial differential equation (3), we chose to solve the mixed first-order-in-time system of equations (5)-(6) for $u_{3}$ and $\mathbf{v}$ in a bounded sub-domain $\Omega$ of $\mathbf{R}^{2}$ (see fig. 4). Working with the mixed first-order formulation instead of the second-order wave equation presents two main advantages. Firstly, it can be coupled with the fictitious domain method ${ }^{29,30}$ for taking into account the free surface boundary condition. Secondly, it enables us to model wave propagation in infinite domains, the case of interest here, by using the Perfectly Matched absorbing Layer $(\mathrm{PML})^{31,32}$. Note that the PML leads to the elimination of reflections from fictitious boundaries such as those which occur in Ref. 18. The fictitious domain method consists in extending the wave propagation problem in a domain $\Omega$ with simple geometry (typically a rectangle in 2D), which enables the use of regular meshes. The free surface boundary condition is then enforced with the introduction of a Lagrange multiplier. This new unknown lives only on the free surface and can be discretized with a non-uniform mesh, different in general from the mesh in the rest of the computation domain (see Fig. 4). 
For the space discretization we use a finite element method, whereas for the time discretization a centered second order finite difference scheme is used. The finite elements are compatible with mass-lumping, which leads to explicit time discretization schemes. For the velocity, we use a new finite element $\operatorname{method}^{33,34}$ and for the pressure we use $P^{1}$ discontinuous functions (this is a different choice from the one in Refs. 33-34)). The Lagrange multiplier is discretized with $P^{1}$ continuous functions. More details on the numerical method can be found in Ref. 29.

The pulse associated with the incident wave is created by a line source of the form

$$
g(\mathbf{x}, t)=F(t) \quad\left(1-\frac{r^{2}}{r_{s}^{2}}\right)^{3} 1_{B_{s}},
$$

where $r$ is the radial coordinate in the sagittal plane. More precisely we take,

$$
F(t)=\left\{\begin{array}{cc}
-2 \pi v_{0}^{2}\left[1-2 \pi v_{0}^{2}\left(t-t_{0}\right)^{2}\right] e^{-\pi^{2} v_{0}^{2}\left(t-t_{0}\right)^{2}} & , t \leq 2 t_{0} \\
0 & , t>2 t_{0}
\end{array},\right.
$$

In the above relations $t_{0}=1 / v_{0}, v_{0}$ is the central frequency of the spectrum of the pulse, and $1_{B_{s}}$ is the characteristic function of the disc $B_{s}$, centered at $\mathbf{x}_{s}$ (location of the source) and with radius $r_{s}$. The function multiplying $F$ in (9) is a smooth approximation to the delta function $\delta(r)$. The radius $r_{s}$ is small, typically a few discretization steps. In our computations we chose $v_{0}=0.50 \mathrm{~Hz}$ and $\mathbf{x}_{s}=(0 \mathrm{~m}, 3000 \mathrm{~m})$. The densities in the bedrock, soft layer and blocks+foundations were chosen to be: $2000 \mathrm{Kg} / \mathrm{m}^{3}, 1300 \mathrm{Kg} / \mathrm{m}^{3}$ and $325 \mathrm{Kg} / \mathrm{m}^{3}$ respectively, whereas the bulk shear wave velocities in these three media were taken to be $600 \mathrm{~m} / \mathrm{s}, 60 \mathrm{~m} / \mathrm{s}$ and $100 \mathrm{~m} / \mathrm{s}$, respectively. The foundation depth of the blocks was $10 \mathrm{~m}$ and the soft layer thickness $50 \mathrm{~m}$. The block widths, heights and separations ranged over 30-60m, 50-70m, and $60-100 m$ respectively (see table 1). Finally, we computed the seismic response of a city with either a single block or no block at all. The media below ground level are the same in all these cases. Most of the aforementioned parameters are close to those of Ref. 25, and are fairly representative of the blocks and the substratum at downtown sites in Mexico City. The 
computational domain was a $3500 m \times 3500 m$ square (see Fig.2) discretized by a grid of 351 nodes in each dimension (more nodes could be taken to get more computational accuracy and a finer description of the motion, notably in the blocks and on the fee surface). This domain was surrounded by a PML layer 30 nodes thick, and 465 nodes where placed on the free surface.

To give a measure of the vulnerability of the blocks of the city, we introduce the vulnerability index $R_{j}$. Let $T$ be the time interval of significant shaking (in the computations this was $240 \mathrm{sec}$ ). We then define $R_{j}$ as the ratio between the time-integral from 0 to $T$ of the modulus squared particle velocity at the midpoint of the top segment of the $j$-th block (located by position vector $\mathbf{x}_{j}$ in fig. 5) and the time integral from $O$ to $T$ of the same quantity measured on the ground (point located by position vector $\mathbf{x}_{0}$ in fig. 5) in the absence of all blocks

$$
R_{j}=\frac{\int_{0}^{T}\left|\partial_{t} u_{3}\left(\mathbf{x}_{j}, t\right)\right|^{2} d t}{\int_{0}^{T}\left|\partial_{t} u_{3}\left(\mathbf{x}_{0}, t\right)\right|^{2} d t}
$$

The subsurface configuration and excitation are the same in the presence and absence of the blocks (other authors ${ }^{35-37}$ have previously employed the denominator of these ratios to quantify ground motion in urban and other areas).

To give a measure of the strength of ground shaking, we introduce the index $R_{j j+1}$ as the ratio between the the time-integral from $O$ to $T$ of the modulus squared particle velocity at the center of the ground segment between the the $j$-th block and the $j+1$-th block (located by position vector $\mathbf{x}_{j j+1}$ in fig. 5) and the time integral from $O$ to $T$ of the same quantity measured on the ground (point located by position vector $\mathbf{x}_{0}$ in fig. 5) in the absence of all blocks

$$
R_{j j+1}=\frac{\int_{0}^{T}\left|\partial_{t} u_{3}\left(\mathbf{x}_{j j+1}, t\right)\right|^{2} d t}{\int_{0}^{T}\left|\partial_{t} u_{3}\left(\mathbf{x}_{0}, t\right)\right|^{2} d t}
$$

\section{Results}


The snapshots of the modulus of the total displacement field in Fig. 6 pertain to the case in which there are no blocks in the city. As mentioned in the introductory remarks, one does not expect the Love modes to be excited to any great extent when the source is far from the layer. This is what is actually observed in Fig. 6 since the displacement field in the soft layer is not typical of that of a Love mode.

Fig. 7 depicts the snapshots relative to a city with a single block. These snapshots indicate a series of hot spots inside the low-velocity layer. This phenomenon betrays the excitation of the fundamental Love mode of the soft layer at the base of the block's foundation. At certain instants, the block displacement also becomes large, but as time goes by, the motion dies away rather quickly in the block, layer and subtratum.

Fig. 8 contains the snapshots for a city with ten blocks. Again, there is an indication of something like a standing wave in the layer betraying the excitation of a quasi-Love mode, which is seen to give rise to rather large response not only in the blocks, but also on the ground, and in the layer (as for the excitation of a pure Love mode). This was also observed in Ref. 25 for a periodic distribution of identical blocks. Here, the variable spacing, irregular sizes and aspect ratios of the blocks spoil the regular standing wave pattern of a pure Love mode or of a quasi-Love mode relative to a periodic structure. Moreover, two successive blocks act in a manner similar to that of the edges of a basin ${ }^{5,6}$ (absent in our model since the lateral extent of the layer is infinite). Indeed, they constitute diffractors whereby Love waves are locally generated, which then presumably travel back and forth in between pairs of blocks, thus resulting in some degree of coupling of the motions of the blocks via the soil so as to result in a duration of the shaking inside the blocks which is apparently longer than the one observed in the one-block configuration (this is substantiated more quantitatively in figs. 10 and 11). Note that the distance between these diffractors (i.e., blocks) is small (several tens of meters) compared to that (of the order of several kilometers) between the edges of the basins which were the object of the studies in Refs. 5 and 41 wherein the interference of waves originating from basin edges was disqualified as being the possible cause of the 
enhanced effects of seismic response in sites such as Mexico City. A more mathematicallyoriented explanation of effects similar to those shown here can be found in Refs. 12,38.

The time records corresponding to figs. 6-8, which are presented in figs. 9-11, call for the following comments.

(i) In fig. 9 it is seen that the configuration with or without the single block produces a response with a relatively short ( $\sim 1 \mathrm{~min})$ duration, this being due essentially to the predominance of radiation damping. This response is not typical of responses in sites in cities such as downtown Mexico City where there exists a rather high density of multiple-story structures and several high-rise buildings. Nevertheless, one aspect of observed response at these locations is present in this figure: the peak motion of the block is larger (here, by a factor of the order 1.8) than the response on the ground in the absence of the block, presumably due to block/soil interaction, i.e., the coupling of the rigid base block mode resonances with the Love mode resonances ${ }^{12,38}$.

(ii) In figs. 10-11, it can be observed that new effects, related to duration lengthening and beating, make their appearance in the response of the 10-building configuration. The duration is much longer than the previous $1 \mathrm{~min}$ at certain locations of this 'city', attaining of the order of $3 \mathrm{~min}$, a remarkable figure considering that the input pulse duration is only a few seconds. Collective causes such as interference and block/soil/block interaction ${ }^{12,38}$ dominate during the first few minutes of shaking and radiation damping dominates the response during the later phase.

(iii) The peak amplitude of block response is not systematically larger at locations of the 10-block city than in the one-block configuration, but the generally-longer duration of response in certain blocks of the 10-block city makes these blocks more vulnerable than the isolated block (due to the fact that the vulnerability index is greater in these blocks than in the isolated block).

(iv) The cumulative response at the top of the blocks varies significantly from one building to another, corresponding to vulnerability indices ranging from $\sim 1.2$ to $\sim 6.7$ for the ten-block set, which suggests that some of the blocks may suffer severe damage, while others will go unscathed, as a result of an earthquake in a city such as this one. 
(v) The (peak and cumulative) response on the ground is not always less at locations on the ground between the buildings than the response in the blocks.

Features (ii)-(v) related to the 10-block configuration are in qualitative agreement with what was observed during earthquakes in urban sites such as Mexico City (see, e.g., Refs. 28, 39).

\section{Discussion}

This work was initiated by the knowledge that $1 \mathrm{D}$ configurations can produce some, but not all, of the response features observed during the recent strong earthquakes in cities built on soft soil such as Mexico City. The traditional (and even quite recent) choice e $^{3,6-}$ 10,16,19,36,40, concerning the analysis of such earthquakes, has been to introduce complexity into the substratum while leaving the free surface flat or with large-scale topographic features. The buildings are not included in such a model and their response is treated separately, using the flat ground motion as the input. This has led to response predictions that are more in line with what has actually been observed during tremors in a variety of cities (Nice, Los Angeles, Mexico City, etc.). Nevertheless, one or several of the features, namely duration, peak velocities and spatial variability, of observed response, differ from those of the predictions ${ }^{3,8,10,40}$. This is possibly due to the fact that the fine structure of the substratum is unknown. Another plausible hypothesis is that small-scale irregularities on the free surface and on the interface between the foundations and the soft soil, introduced by the existence of buildings in a city, may contribute significantly to the overall motion of the site.

We have tested this hypothesis using a simple 1D-like model of the substratum, a fairly-realistic model of the buildings (modeled as homogenized blocks) in a city, and a comprehensive wave/structure interaction model, i.e., one that can account for many degrees of freedom of motion of the blocks and soil (although constrained to anti-plane motion in an elastic medium), as well as for all block/soil and block/soil/block interactions. An example of ten different non equally-spaced blocks indicates that the latter play an essential role in exciting a quasi-Love mode which, when coupled to the modes of the blocks, make these blocks shake with relatively large amplitude over a very long period. These couplings are surely mediated by block/soil/block interactions and interference effects so that it is not appropriate to base a description of the seismic response in a city on that of only one of its components (i.e., a single block). On the other hand, the explicit inclusion of more than one buildings into the theoretical model reduces the risk of not accounting for the collective 
effects that are apparently an essential ingredient of the earthquake responses in an urban area. A similar conclusion was proposed in Refs. 25 and 28. Also, a series of other numerical results not shown herein, obtained by our numerical scheme, has shown that the collective effects of beating, spatial variability, very long durations associated with large vulnerability indices, at certain ground and block locations, are also present in the seismic response of quasi-periodic cities (i.e., ones with the same set of dissimilar blocks as previously, but whose spacing is constant) for blocks whose (constant) spacing ranges from $30 \mathrm{~m}$ to $120 \mathrm{~m}$. This gives further support to the hypothesis that the anomalous seismic response in cities built on soft soil is largely due to the presence of the (many) buildings therein, whatever (within reasonable limits) be the sizes, aspect ratios and separations of these structures.

These findings will have to be substantiated by further computations with continuum or other exact models to account for viscoelastic effects, in-plane motion, and 3D cities. In addition, it will be necessary to examine to what extent anomalous response is affected by the location and type of seismic source as well as by the duration of the pulse radiated from this source. Work along these lines is in progress in our laboratory and will be the subject of forthcoming publications.

\section{Acknowledgements}

This research was carried out within the framework of the Action Concertée Incitative "Prévention des Catastrophes Naturelles" entitled "Interaction 'site-ville' et aléa sismique en milieu urbain" of the French Ministry of Research.

\section{References}

[1] EQE: The January 17, 1995 Kobe Earthquake, EQE Summary Report, April 1995, http://www.eqe.com/publications/kobe/kobe.htm

[2] Mucciarelli M., Camassi R. \& Gallipoli M.R.: The Izmit (Turkey) 1999 earthquake, http://www.pz.cnr.it/imaaa/turchia/report_e.html

[3] Semblat J.-F., Duval A.-M. \& Dangla P.: Numerical analysis of seismic wave amplification in Nice (France) and comparisons with experiments. Soil Dynam.Earthqua.Engrg. 19, 347-362, 2000. 
[4] Singh S.K., Mori A., Mena E., Krüger F. \& Kind R. : Evidence for anomalous body-wave radiation between 0.3 and $0.7 \mathrm{~Hz}$ from the 1985 september 19 Michoacan, Mexico earthquake, Geophys.J.Int. 101, 37-48, 1990.

[5] Chavez-Garcia F.J. \& Bard P.-Y.: Site effects in Mexico City eight years after the September 1985 Michoacan earthquakes, Soil Dyn.Earthquake Engrg. 13, 229-247 , 1994.

[6] Bard P.-Y., Eeri M., Campillo M., Chavez-Garcia F.J. \& Sanchez-Sesma F.J.: The Mexico earthquake of September 19, 1985--a theoretical investigation of large-and smallscale amplification effects in the Mexico City valley, Earthquake Spectra, 4, 609-633, 1988.

[7] Olsen K.B.: Site Amplification in the Los Angeles Basin from Three-Dimensional Modeling of Ground Motion, Bull.Seism.Soc.Am., 90, 77 - 94, 2000.

[8] Hartzell S., Cranswick E., Frankel A., Carver D. \& Meremonte M.: Variablility of site response in the Los Angeles urban area, Bull.Seism.Soc.Am., 87, 1377-1400, 1997.

[9] Wald D.J. \& Graves R.W.: The seismic response of the Los Angeles Basin, California, Bull.Seism.Soc.Am., 88, 337-356, 1998.

[10] Wald L.A. \& Mori J.: Evaluation of Methods for Estimating Linear Site-Response Amplificatons in the Los Angeles Region, Bull.Seism.Soc.Am., 90, 32-41, 2000.

[11] Burridge R. : Soil amplification of plane seismic waves, Phys.Earth Planet Int., 22, 122$136,1989$.

[12] Wirgin A.: Love waves in a slab with rough boundaries, in Recent Developments in Surface Acoustic Waves, Parker D.F. \& Maugin G.A. (eds.), Springer, Berlin, 145-155, 1988.

[13] Wirgin A. : Resonant response of a soft semi-circular cylindrical basin to a SH sismic wave, Bull.Seism.Soc.Am., 86, 914-920, 1996.

[14] Hill N.R. \& Levander A.R.: Resonances of low-velocity layers with lateral variations, Bull.Seism.Soc.Am. 74, 521-537, 1984.

[15] Sanchez-Sesma F.J. \& Esquivel J.A.: Ground motion on alluvial valleys under incident plane SH waves, Bull.Seism.Soc.Am., 69, 1107-1120, 1979.

[16] Campillo M., Sanchez-Sesma F.J. \& Aki K. : Influence of small lateral variations of a soft surficial layer on seismic ground motion, Soil Dyn.Earthquake Engrg., 9, 284-287, 1990.

[17] Bard P.-Y. \& Bouchon M.: The two-dimensional resonance of sediment-filled valleys, Bull.Seism.Soc.Am., 75, 519-541, 1985. 
[18] Levander A.R. \& Hill N.R.: P-SV resonances in irregular low-velocity surface layers, Bull.Seism.Soc.Am. 75, 847-864, 1984.

[19] Avilés J. \& Pérez-Rocha L.E. : Site effects and soil-structure interaction in the valley of Mexico, Soil Dynam.Earthqua.Engrg., 17, 29-39, 1998.

[20] Trifunac, M.D.: Interaction of a shear wall with the soil for incident plane SH waves. Bull.Seism.Soc.Am. 62, 63-83, 1972.

[21] Luco J.E. \& Contesse L. : Dynamic structure-soil-structure interaction, Bull. Seism. Soc. Am. 63, 1289-1303, 1973.

[22] Wong H.L. \& Trifunac M.D.: Two-dimensional, antiplane, building-soil-building interaction for two or more buildings and for incident plane SH waves. Bull.Seism.Soc.Am. 65, 1863-1885, 1975.

[23] Todorovska M.I. \& Trifunac M.D., Antiplane earthquake waves in long structures, J.Engrg.Mech. 115, 2687-2708, 1989.

[24] Wolf J.P.: Vibration Analysis Using Simple Physical Models, Prentice-Hall, Englewood Cliffs, 1994.

[25] Wirgin A. \& Bard P.-Y.: Effects of buildings on the duration and amplitude of ground motion in Mexico City, Bull.Seism.Soc.Am., 86 , 914-920, 1996.

[26] Clouteau, D. \& Aubry, D.: Modifications of the ground motion in dense urban areas. J.Comput.Acoust. 9, 1659-1675, 2001.

[27] Chiaruttini C., Grimaz S. \& Priolo E. : Modeling of ground motion in the vicinity of massive structures, Soil Dynam.Earthqua.Engrg. 15, 75-82, 1996.

[28] Gueguen P., Bard P.-Y. \& Chavez-Garcia F.J. : Site-city seismic interaction in Mexico City like environments : an analytic study, Bull.Seism.Soc.Am. 92, 794-804, 2002 .

[29] Tsogka C., Modélisation mathématique et numérique de la propagation des ondes élastiques tridimensionnelles dans des milieux fissures. Thèse de Doctorat, Université Paris IX Dauphine, Paris, 1999.

[30] Bécache E., Joly P. \& Tsogka C.: Application of the fictitious domain method to 2D linear elastodynamic problems. J.Comput.Acoust. 9, 1175-1202, 2001.

[31] Bérenger J.P. A perfectly matched layer for the absorption of electromagnetic waves. J.Comput.Phys. 114, 185-200, 1994.

[32] Collino F. \& Tsogka C.: Application of the PML absorbing layer model to the linear elastodynamic problem in anisotropic heterogeneous media, Geophys. 66, 294-307, 2001. 
[33] Bécache E., Joly P. \& Tsogka C.: Etude d'un nouvelle élément fini mixte permettant la condensation de masse. C.R.Acad.Sci.Paris Sér.I Math. 324, 1281-1286, 1997.

[34] Bécache E., Joly P. \& Tsogka C.: An analysis of new mixed finite elements for the approximation of wave propagation problems. SIAM J.Numer.Anal. 37, 1053-1084, 2000.

[35] Fäh D. \& Panza G.F., Realistic modeling of observed seismic motion in complex sedimentary basins, Annal.Geofis. 37, 1771-1797, 1994.

[36] Olsen K.B., Archuleta R.J. and Matarese J.R.: Three-dimensional simulation of a magnitude 7.75 earthquake on the San Andreas fault, Science, 270, 1628-1632, 1995.

[37] Trifunac M.D. \& Brady A.G.: A study on the duration of strong earthquake ground motion, Bull.Seism.Soc.Am., 65, 1975, 581-626.

[38] Wirgin A. \& Kouoh-Bille L. : Amplification du mouvement du sol au voisinage d'un groupe de montagnes de profil rectangulaire ou triangulaire soumis à une onde sismique SH, in Génie Parasismique et Aspects Vibratoires dans le Génie Civil, AFPS, SaintRémy-lès-Chevreuse, ES-28--ES-37, 1993.

[39] PEER, Long-duration soil motions recorded at the TACY station in Mexico City during the 1985 Michoacan earthquake, http://peer.berkeley.edu/research/motions/.

[40] Olsen K.B. \& Archuleta R.J.: 3D simulation of earthquakes on the Los Angeles fault system, Bull.Seism.Soc.Am., 86, 575-596, 1996.

[41] Chavez-Garcia F.J., Ramos-Martinez J. \& Romero-Jimenez E.: Surface-Wave dispersion analysis in Mexico City, Bull.Seism.Soc.Am., 85, 1116-1126. 


\begin{tabular}{|c|c|c|c|c|c|c|c|c|c|c|}
\hline Building & $\boldsymbol{1}$ & $\mathbf{2}$ & $\mathbf{3}$ & $\mathbf{4}$ & $\mathbf{5}$ & $\mathbf{6}$ & $\mathbf{7}$ & $\boldsymbol{8}$ & $\mathbf{9}$ & $\mathbf{1 0}$ \\
\hline Height $\boldsymbol{h}$ & 50 & 50 & 50 & 60 & 60 & 60 & 70 & 70 & 70 & 70 \\
\hline Width $\boldsymbol{w}$ & 30 & 40 & 50 & 30 & 40 & 50 & 30 & 40 & 50 & 60 \\
\hline $\begin{array}{c}\text { Separation } \\
\boldsymbol{d}(\boldsymbol{i}, \boldsymbol{i}+\mathbf{1})\end{array}$ & 70 & 90 & 60 & 80 & 100 & 60 & 90 & 80 & 60 & \\
\hline
\end{tabular}

Table 1 Geometrical parameters $h, w, d$ of the ten-block idealized city. The units are meters. 


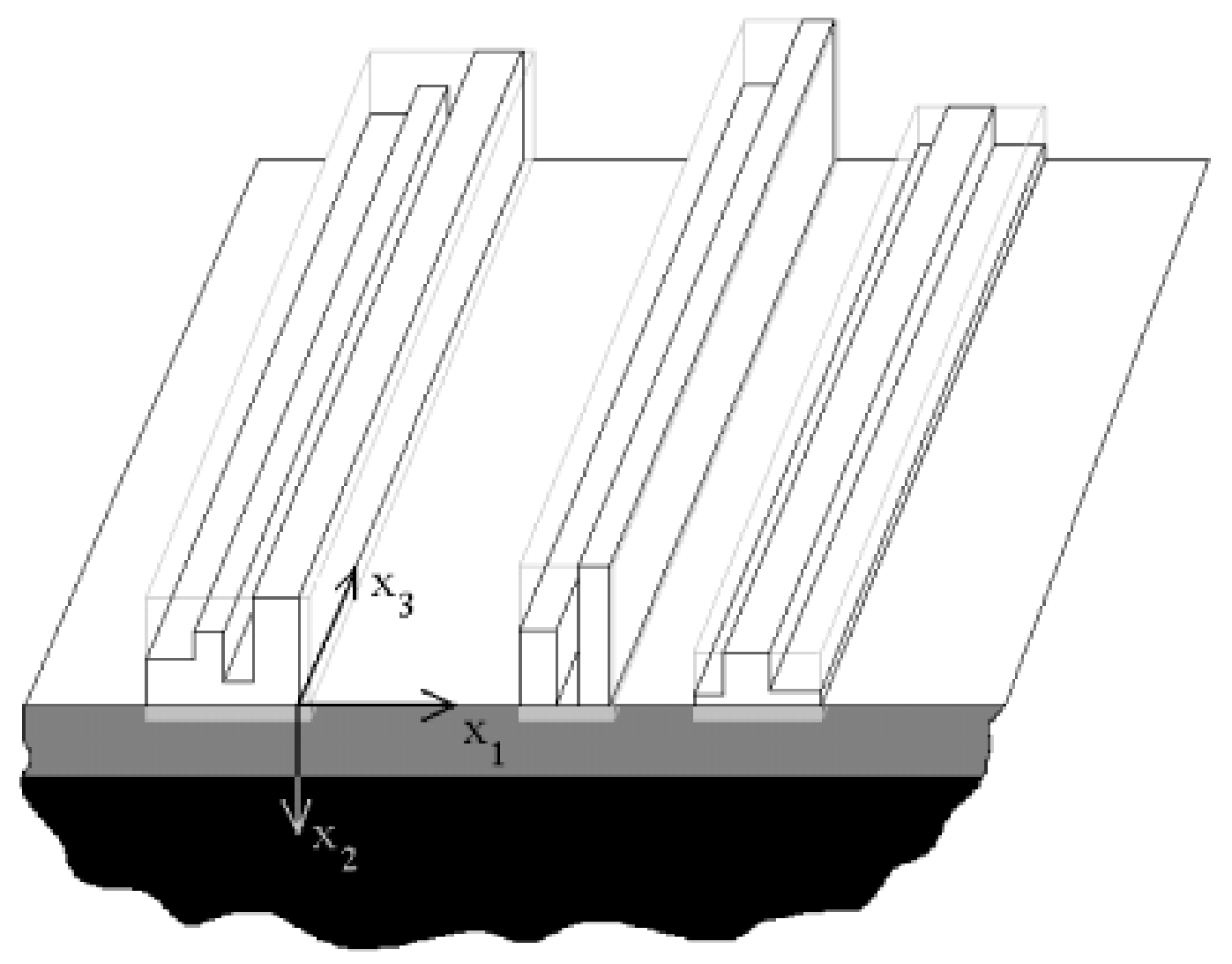

Fig. $13 \mathrm{D}$ view of the idealized city. The black region is the hard rock basement in which is imbedded the line source (parallel to the $x_{3}$ axis and located at a certain depth, on the $x_{2}$ axis) responsible for the seismic wave, the dark grey region is the soft layer in which the blocks, enclosed in the light grey parallelepipeds, are anchored. A block can be composed of one or several substructures (e.g., buildings, all invariant with respect to $x_{3}$ in terms of geometry and composition). In the absence of the blocks the ground is the plane $x_{2}=0$. The sagittal (cross-section) plane is the $x_{1}-x_{2}$ plane. In this plane, a given block appears to be heterogeneous, due to its composite nature. In the analysis, each block is homogenized, i.e., replaced by a homogeneous block which gives rise to approximately the same average response as that of the heterogeneous block. 


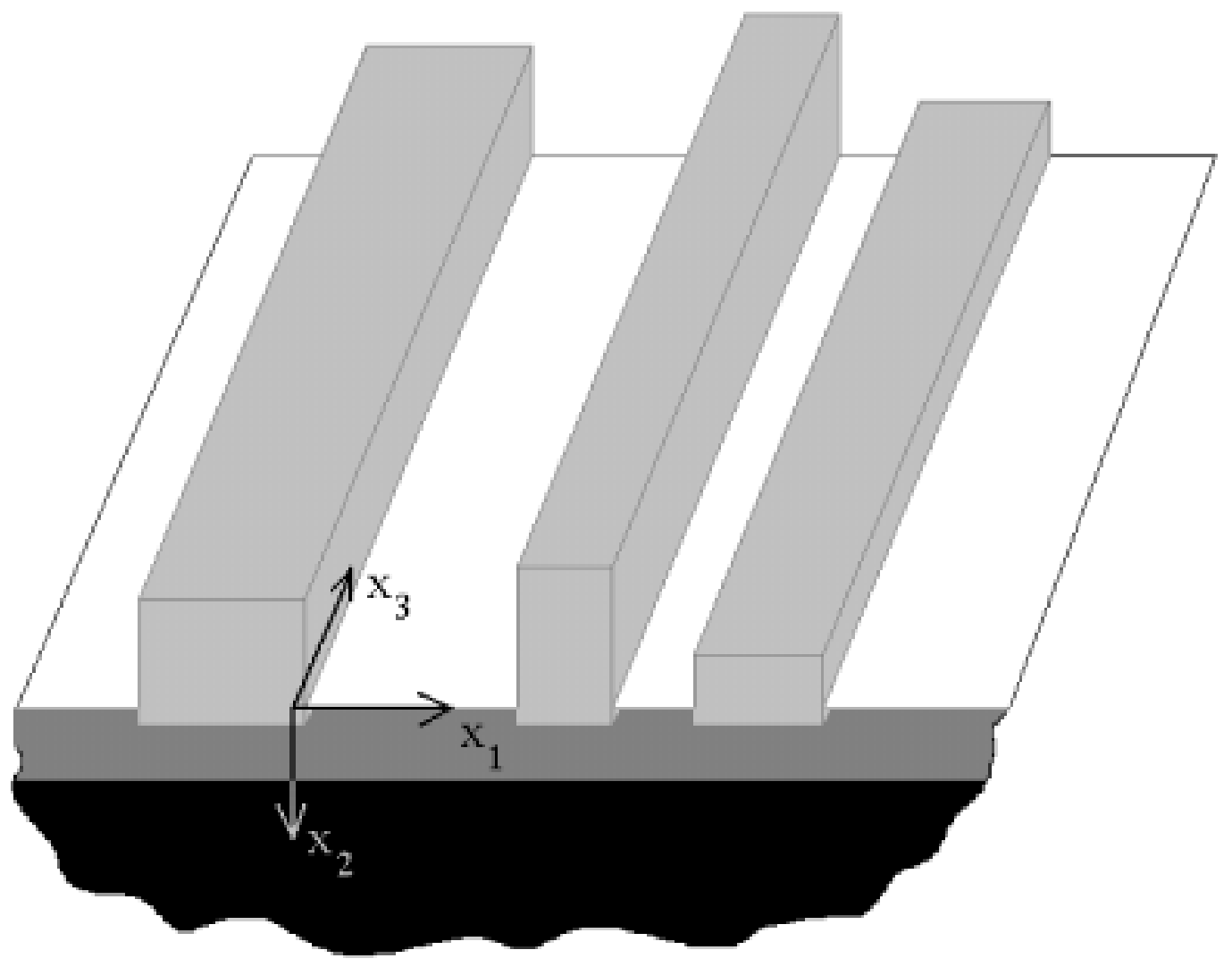

Fig. 2 Homogenized version of the configuration of fig.1. The homogeneous compositions of the different blocks are not necessarily identical but assumed so in the computations. Note that the blocks are partially imbedded in the soft layer. The soft layer and hard basement are assumed to be homogeneous at the outset. 


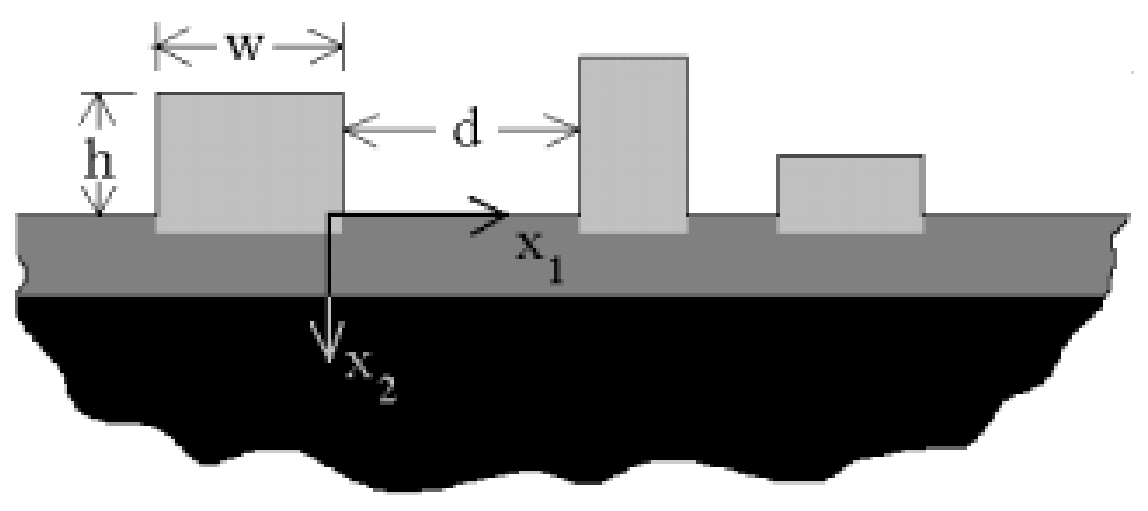

Fig. 3 Sagittal plane view of the idealized city with homogenized blocks. The height, width, and separation of the blocks are denoted by $h, w$, and $d$ respectively. These are generally different from block to block. 


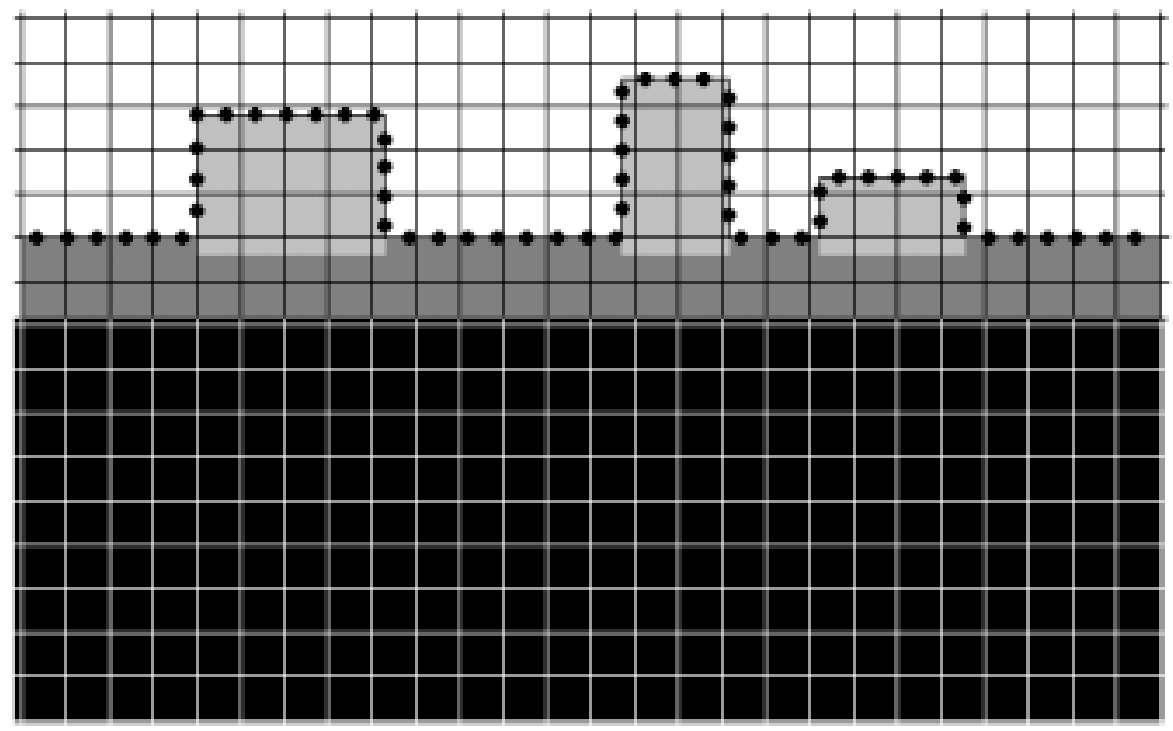

Fig. 4 Schematic view of the grid used in the bedrock/soft layer/vacuum rectangular region $\Omega$ (discretized by a regular network of horizontal and vertical lines) and on the free surface (segments with dots). The nodal points on the free surface do not necessarily coincide with nodal points of the bedrock/soft layer/vacuum region. The number of points in both of these sets is considerably larger in the actual computations than in this drawing. 


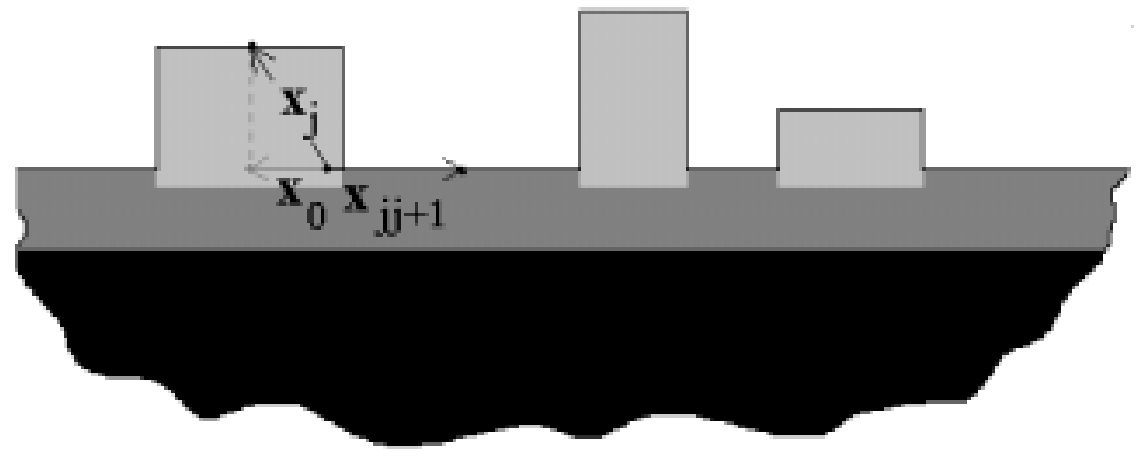

Fig. 5 Schematic view of the vectors $\mathbf{x}_{0}, \mathbf{x}_{j}, \mathbf{x}_{j j+1}$ which define the positions of a point on the free surface in the absence of the buildings (i.e., on flat ground), the midpoint of the top segment of the $j$-th block, and the midpoint of the ground segment between the $j$-th and $j+1$-th blocks, respectively. The vulnerability indices are computed at these three points. 


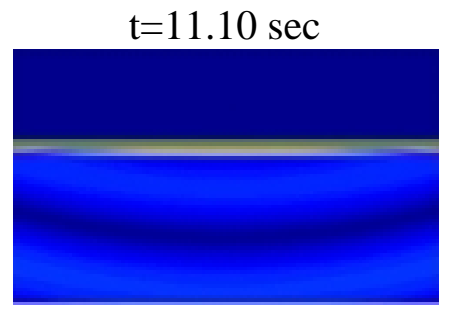

$\mathrm{t}=16.65 \mathrm{sec}$

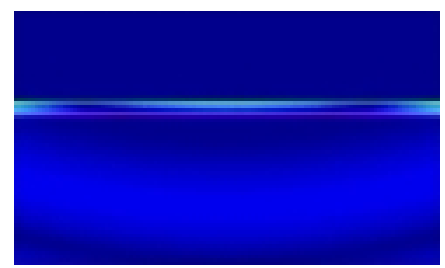

$\mathrm{t}=22.20 \mathrm{sec}$

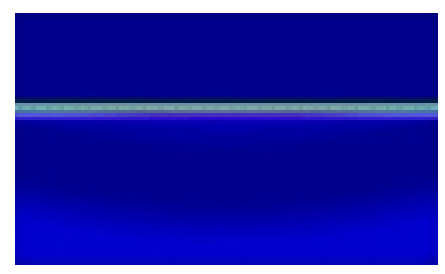

$$
\mathrm{t}=27.75 \mathrm{sec}
$$

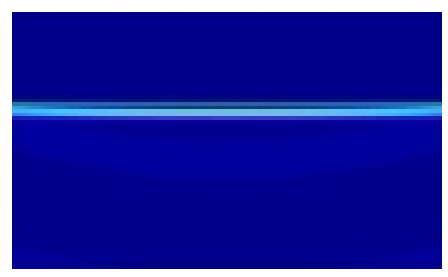

$\mathrm{t}=12.95 \mathrm{sec}$

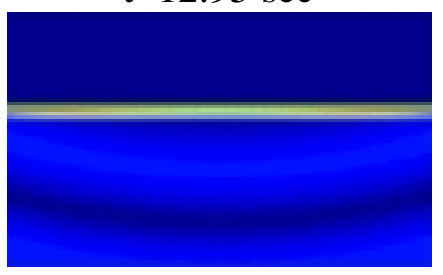

$\mathrm{t}=18.50 \mathrm{sec}$

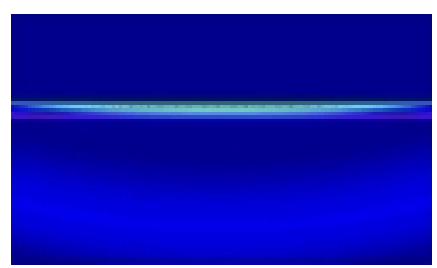

$\mathrm{t}=24.05 \mathrm{sec}$

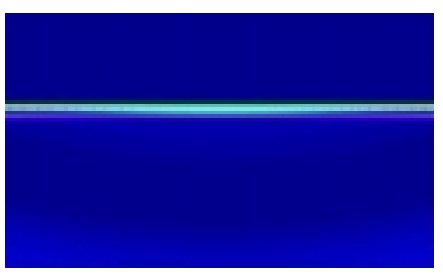

$t=29.60 \mathrm{sec}$

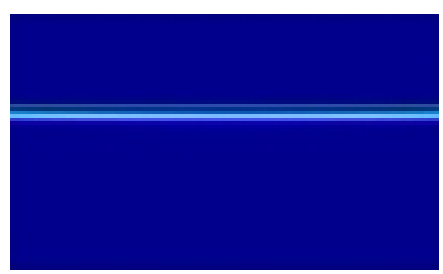

$\mathrm{t}=14.80 \mathrm{sec}$

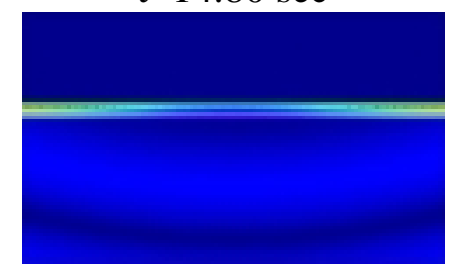

$\mathrm{t}=20.35 \mathrm{sec}$

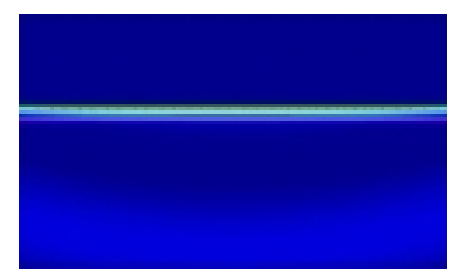

$\mathrm{t}=25.90 \mathrm{sec}$

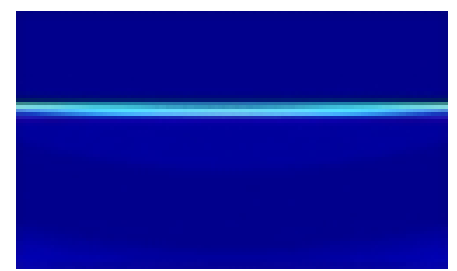

$\mathrm{t}=31.45 \mathrm{sec}$

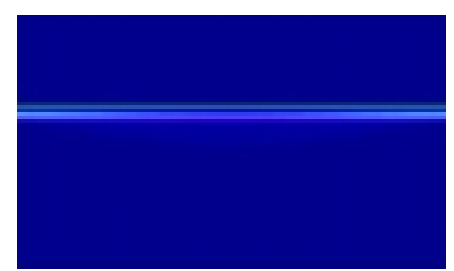

Fig. 6 Snapshots of the total (i.e., incident plus scattered) displacement field, at various instants $t$, for a 'city' in the absence of all blocks. Red designates large displacement and blue small displacement. Time is measured in seconds. 


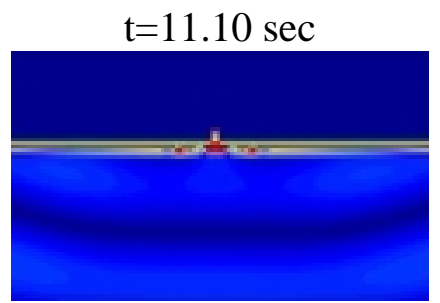

$\mathrm{t}=16.65 \mathrm{sec}$

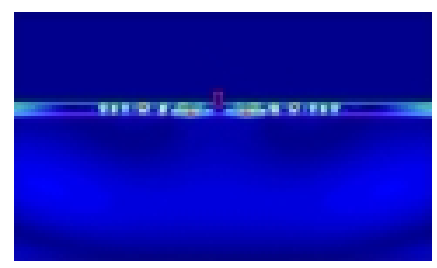

$\mathrm{t}=22.20 \mathrm{sec}$

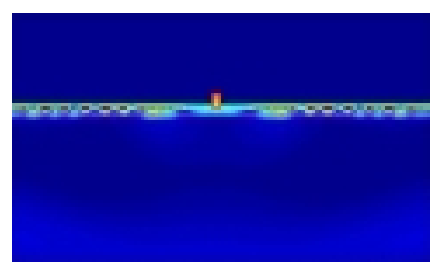

$t=27.75 \mathrm{sec}$

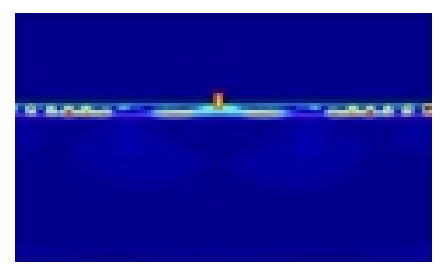

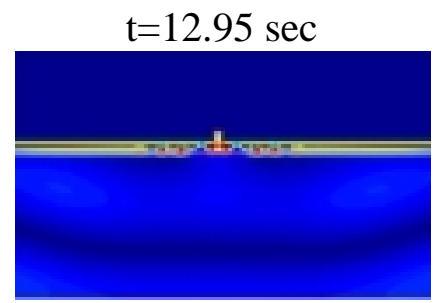

$\mathrm{t}=18.50 \mathrm{sec}$

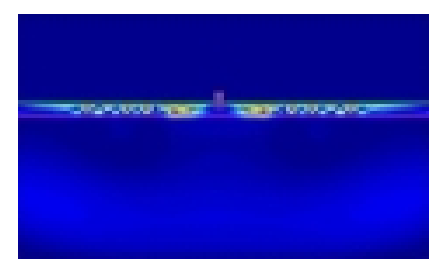

$t=24.05 \mathrm{sec}$

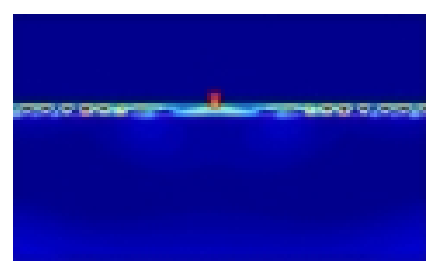

$t=29.60 \mathrm{sec}$

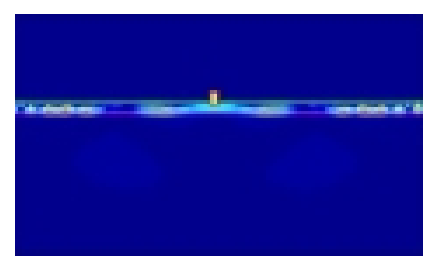

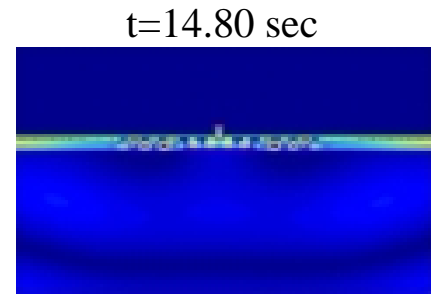

$\mathrm{t}=20.35 \mathrm{sec}$

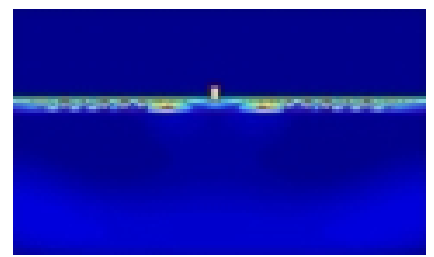

$t=25.90 \mathrm{sec}$

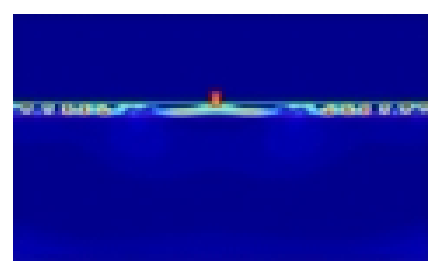

$\mathrm{t}=31.45 \mathrm{sec}$

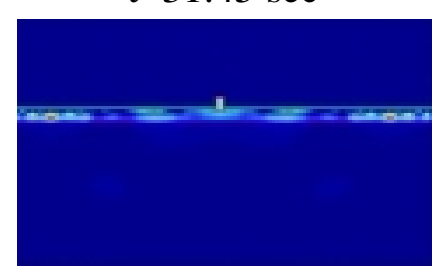

Fig. 7 Snapshots of the total (i.e., incident plus scattered) displacement field, at various instants $t$, for a 'city' composed of one block. Red designates large displacement and blue small displacement, with the color scale being the same as in fig. 6. Time is measured in seconds. 


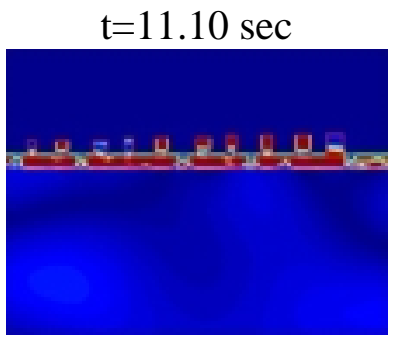

$t=16.65 \mathrm{sec}$

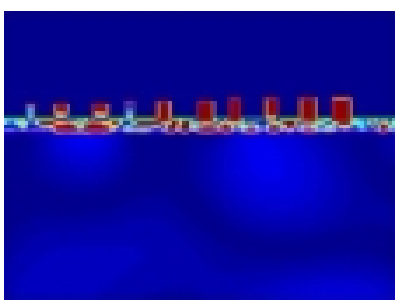

$\mathrm{t}=22.20 \mathrm{sec}$

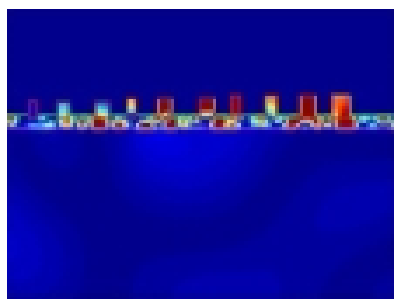

$\mathrm{t}=27.75 \mathrm{sec}$

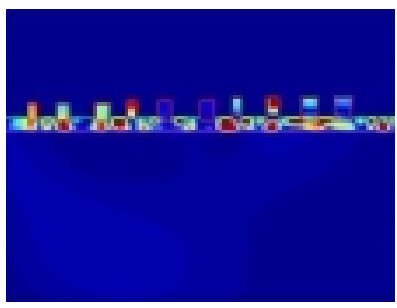

$\mathrm{t}=12.95 \mathrm{sec}$

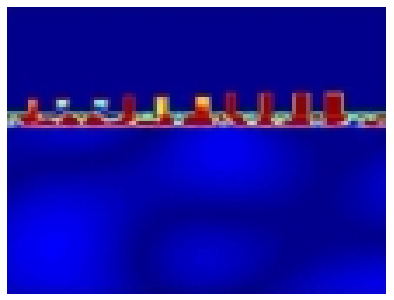

$\mathrm{t}=18.50 \mathrm{sec}$

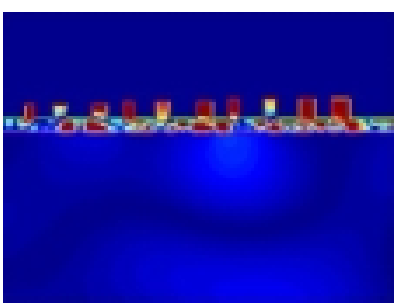

$\mathrm{t}=24.05 \mathrm{sec}$

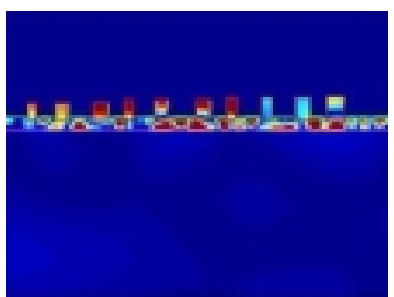

$t=29.60 \mathrm{sec}$

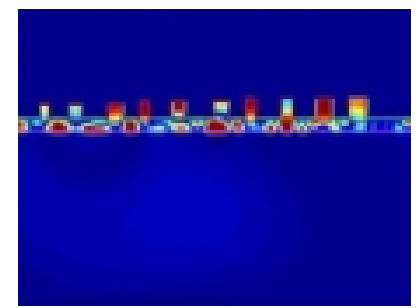

$\mathrm{t}=14.80 \mathrm{sec}$

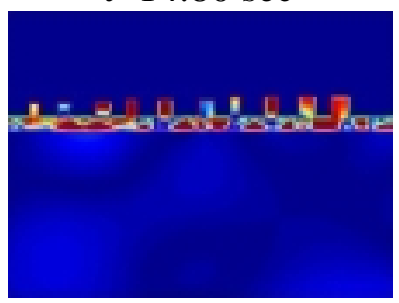

$\mathrm{t}=20.35 \mathrm{sec}$

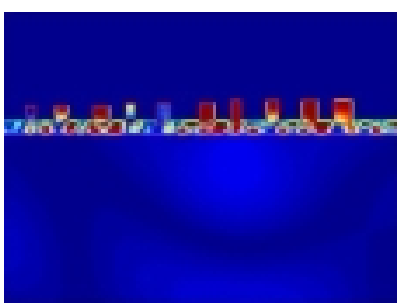

$t=25.90 \mathrm{sec}$

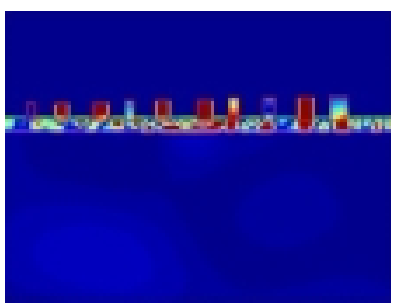

$\mathrm{t}=31.45 \mathrm{sec}$

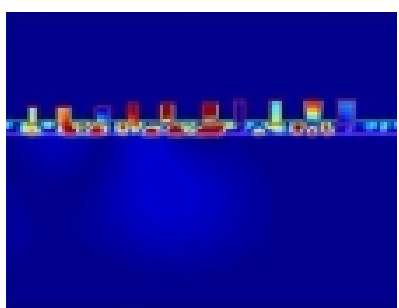

Fig. 8 Snapshots of the total (i.e., incident plus scattered) displacement field, at various instants $t$, for a 'city' composed of ten different blocks with different spacings. Red designates large displacement and blue small displacement, with the color scale being the same as in fig. 6. Time is measured in seconds. 
$R=1.7960$

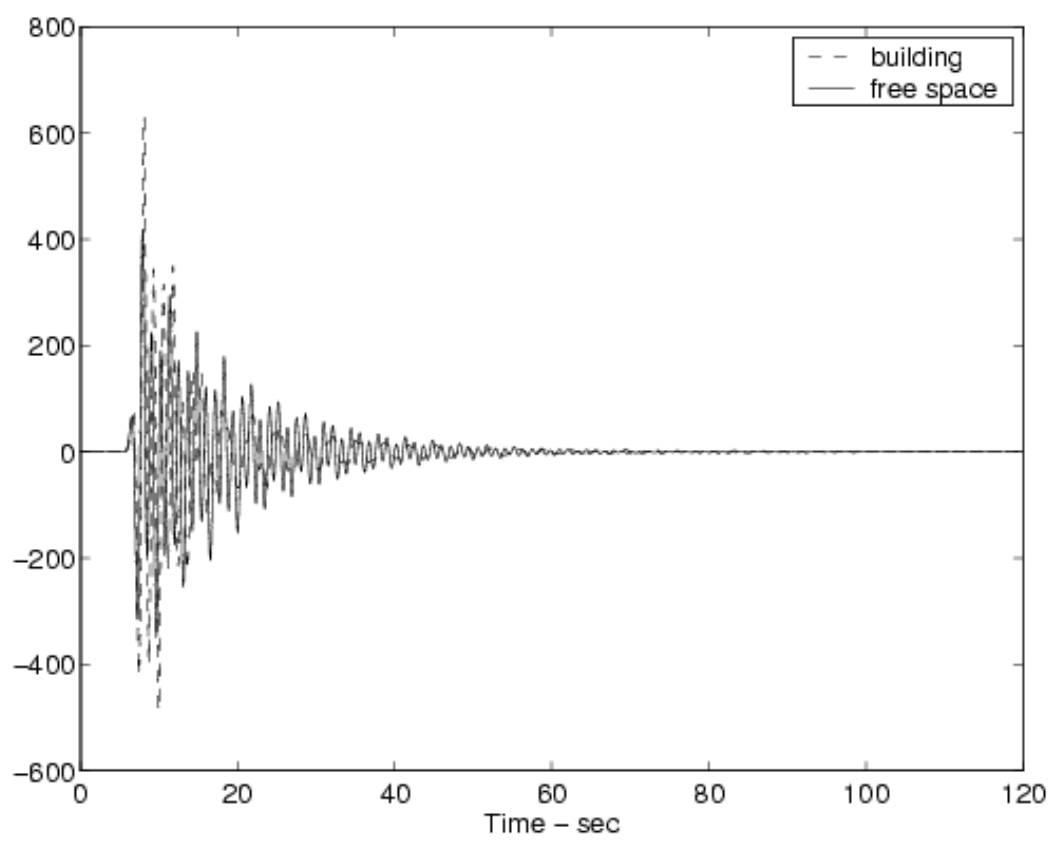

Fig. 9 Time records of total particle velocity for a 'city' with only one block ( $h=50 \mathrm{~m}$, $w=30 \mathrm{~m})$. The two curves correspond to the particle velocity at ground level in the absence of the block (solid curve; same configuration as in fig. 6) and at the center of the top of the block (dashed curve; same configuration as in fig. 7). 


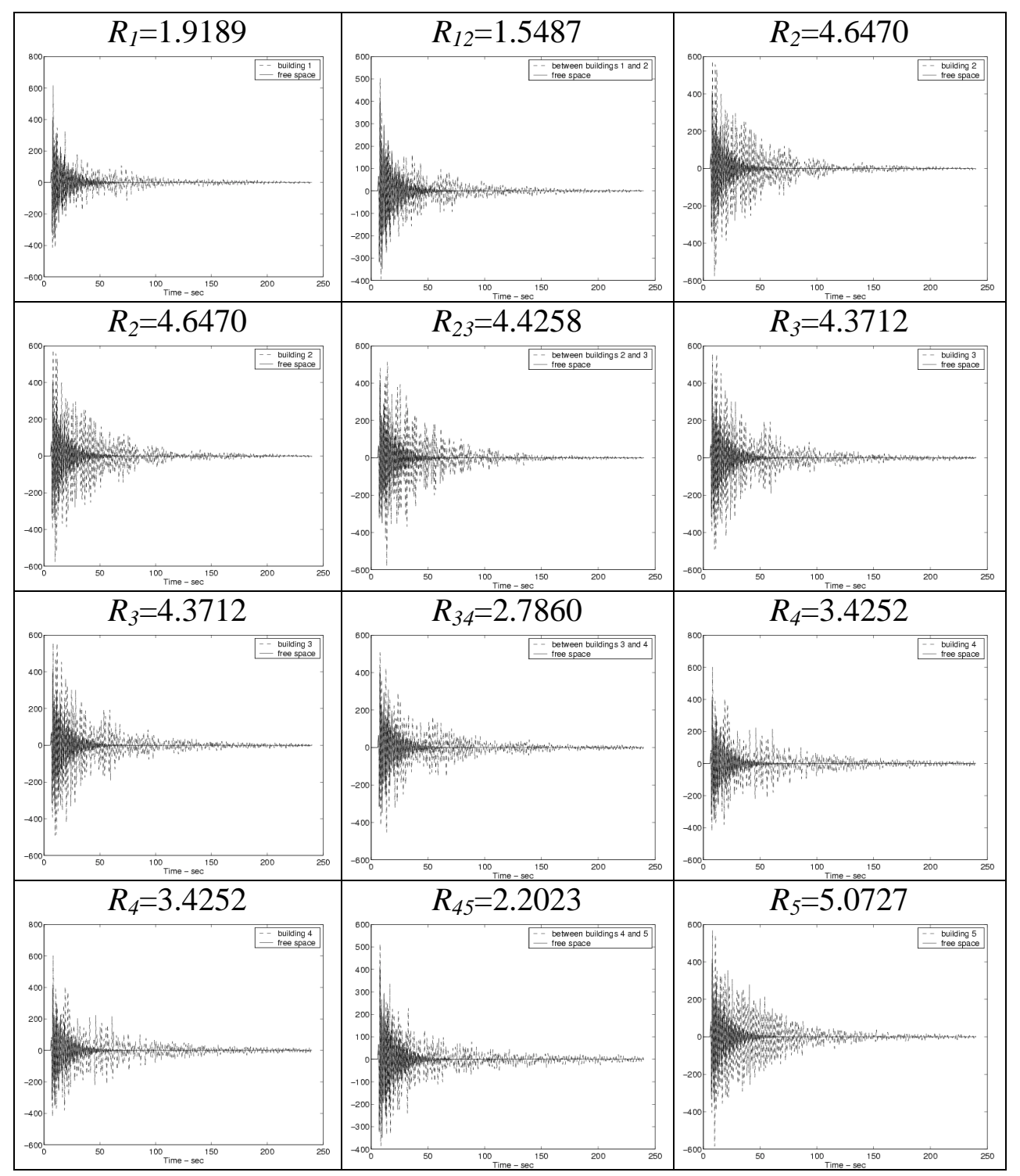

Fig. 10 Time records of total particle velocity for a 'city' with ten blocks having different spacings (same configuration as in fig. 8). Each row of the figure depicts the particle velocity (in $\mathrm{m} / \mathrm{sec}$ ): at the center of the top of the $j$-th block (left), the center of the ground segment between the $j$-th and $(j+1)$-th block (middle) and the $(j+1)$-th block (right). The solid curves in all the subfigures represent the particle velocity at ground level in the absence of blocks. The vulnerability indices $R_{j}$ at the top of the $j$-th block and $R_{j j+1}$ on the ground between the $j$ th and $j+1$-th blocks, are indicated at the top of each subfigure. The abscissas designate time, and range from 0 to $250 \mathrm{sec}$. Note that the scales of the ordinates vary from one subfigure to another. 


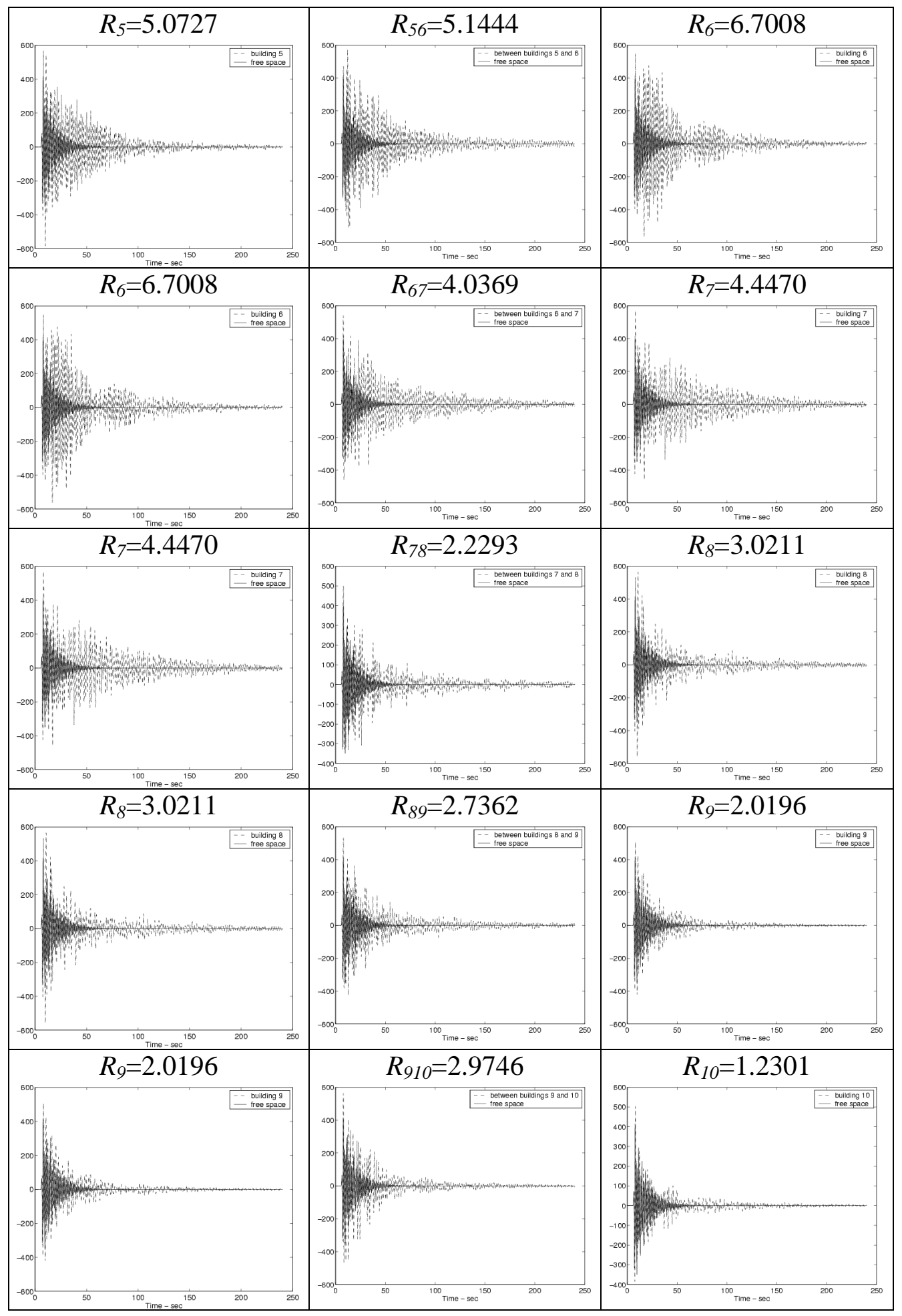

Fig. 11 Same as Fig. 10, for the remainder of the blocks of the 10-block city. 